\title{
Investigation of Hot-Spots in TomoDirect 3DCRT Breast Treatment
}

\author{
Quan Chen1,2*, Matthew Mallory',3, Edwin Crandley ${ }^{2,4}$, Shiv Khandelwal2 \\ ${ }^{1}$ Department of Radiation Medicine, University of Kentucky, Lexington, USA \\ ${ }^{2}$ Department of Radiation Oncology, University of Virginia, Charlottesville, USA \\ ${ }^{3}$ Department of Radiation Oncology, University of Kansas Cancer Center, Kansas City, USA \\ ${ }^{4}$ Department of Radiation Oncology, Eastern Virginia Medical School, Norfolk, USA \\ Email: *qchen@uky.edu
}

How to cite this paper: Chen, Q., Mallory, M., Crandley, E. and Khandelwal, S. (2018) Investigation of Hot-Spots in TomoDirect 3DCRT Breast Treatment. International Journal of Medical Physics, Clinical Engineering and Radiation Oncology, 7, 376-390. https://doi.org/10.4236/ijmpcero.2018.73031

Received: June 23, 2018

Accepted: August 17, 2018

Published: August 20, 2018

Copyright $\odot 2018$ by authors and Scientific Research Publishing Inc. This work is licensed under the Creative Commons Attribution International License (CC BY 4.0).

http://creativecommons.org/licenses/by/4.0/

\begin{abstract}
Background: Conventional tomotherapy platforms only allow for the delivery of helical IMRT. However the use of IMRT and helical delivery in breast cancer treatment is non-standard. Newer tomotherapy units are equipped with a static-beam mode with 3DCRT capabilities. During the clinical use, we frequently observe hot-spots in the plan that renders the plan clinically unacceptable. The purpose of this study is to investigate the underlying cause of the hot-spots in tomotherapy static-beam breast treatment and possible solutions. Materials/Methods: Theories about the formation of the hot-spot were developed. Eight lumpectomy patients contoured according to RTOG-1005 specifications were also used to illustrate the magnitude of hot-spots under various planning strategies. Two tangential beams were used for the whole breast irradiation plan with prescription dose of $40 \mathrm{~Gy}$ in 15 fractions. Results: The hot-spot was identified as the behavior of the optimization engine when part of the target region was blocked. With the current design of tomotherapy's 3DCRT planning where user adjustment was greatly limited, none of the planning strategies were able to reduce the hot-spots to acceptable levels in the eight patients studied. The best strategy still produced an average of 48.5 Gy (121\% of prescription dose) hot-spot dose and $30.4 \mathrm{cc}$ hot-spot volume (volume receiving $>110 \%$ prescription dose). It is also shown that the hot-spot was not a result of energy or other physical limitation of the radiation device. By manually adjusting the plan sinogram, the maximum hot-spot dose drops from $121 \%$ to $111 \%$ and the hot-spot volume drops from $30 \mathrm{cc}$ to $6 \mathrm{cc}$ on average. Conclusions: While TomoDirect 3DCRT showed great promise in breast treatment, treatment planning software improvements may be needed in order to improve the clinical acceptability by reducing hot-spots in normal tissue.
\end{abstract}




\section{Keywords}

TomoTherapy, TomoDirect, Accelerated Whole Breast Irradiation, 3DCRT, RTOG-1005

\section{Introduction}

Breast conserving surgery with adjuvant radiotherapy offers equal survival to mastectomy for T1-2 N0 breast cancer [1]-[7], and current methods of postlumpectomy whole breast irradiation (WBI) result in local control rates of up to 95\% [8] [9]. For WBI, 3-dimensional conformal radiation therapy (3DCRT) plans are typically used with opposed tangential beams in order to avoid the contralateral breast and thoracic organs [10]. Compared to 3DCRT, IMRT has demonstrated improved dose distributions [11] [12] [13] and reduced acute radiation dermatitis [14] [15] in breast cancer patients. However, the use of inverse IMRT in breast cancer has not been demonstrated to improve late toxicity or oncologic outcomes [16]. Therefore, inverse IMRT remains non-standard and is often not approved by insurers unless required for cardiac avoidance [17].

Tomotherapy (Accuray Inc., Sunnyvale, CA) is a unique treatment platform that translates the patient through a ring gantry and delivers fan-beam radiation with a 6-MV linear accelerator in a helical fashion [18]. Tomotherapy is designed for intensity modulation, with features such as a slit beam and fast binary multi-leaf-collimator (MLC) enable it to generate homogeneous target coverage [19]. However, helically delivered radiation is inefficient in breast irradiation because optimal tangential beam angles occupy only a small fraction of the 360-degree revolution. In addition, integral dose to normal tissues is increased [20]. These limitations, coupled with the reimbursement issues surrounding IMRT in breast cancer, have presented obstacles for tomotherapy to be an all-in-one treatment unit.

In order to overcome the major limitations of conventional tomotherapy in breast cancer, newer tomotherapy units are equipped with static delivery mode and a 3DCRT plan mode. The static-beam modes were assigned the name TomoDirect, and up to 12 static-beam angles may be used in one plan. Both the static-beam and 3DCRT modes were aimed to resolve the obstacles mentioned above for breast treatment.

Because tomotherapy is intensity modulated by design, the 3DCRT modes of tomotherapy are actually a crippled form of IMRT in disguise. Similar to the inverse-optimized 3DCRT techniques developed for conventional linac [21], tomotherapy 3DCRT plan was created by the same fluence optimization algorithm used by tomotherapy IMRT. The only difference is that in 3DCRT mode, users can no longer specify optimization constraints to specific structures. Instead, users are provided simple operations such as selection of a target and the percentage of the target covered by the prescription dose, assigning blocking structures, 
choosing between high and low "compensation" which determines the modulation factor, and switching on/off "normal tissue homogeneity" which puts a preset penalty on hot-spot. Because of the inherent IMRT design and simple interface, we have found in our clinical practice that tomotherapy 3DCRT plans can often attain IMRT-like plan quality without labor-intensive subfield modifications used by the forward planning in traditional linear accelerators.

However, we frequently observed unacceptable healthy tissue hot-spots when we use static-beam 3DCRT for breast planning on tomotherapy. This has forced us to abandon the static-beam 3DCRT mode for breast planning all together. The same issue has been reported by other tomotherapy users [10] [20] [22]. These hot-spots have been speculated to occur as a result of the relatively soft 6 MV beam [10] [20]. However, during our clinical practice, we have observed severe hot spots in normal sized patients. Therefore, it is less likely that the soft 6 MV spectrum was the culprit. In this study, we attempt to investigate the underlying cause of the hot-spots in tomotherapy static-beam breast treatment and to find possible solutions.

\section{Methods and Materials}

Figure 1 illustrates the contouring and beam arrangement commonly used for static-beam 3DCRT planning. Contours were created by a radiation oncologist according to RTOG-1005 [23] specifications on VelocityAI software (Velocity Medical Solutions). Due to the tight limit on contralateral breast dose (maximum dose $<3.84 \mathrm{~Gy}[23]$ ), the entire contralateral breast was used as a complete block structure. In addition, a series of "LungHeartBlocking" structures were created for exploring the desired target coverage/OAR sparing tradeoff. The blocking structure were created by taking a union of the ipsilateral lung and heart, then cropping the breast_PTV expanded by various margins. As we trying

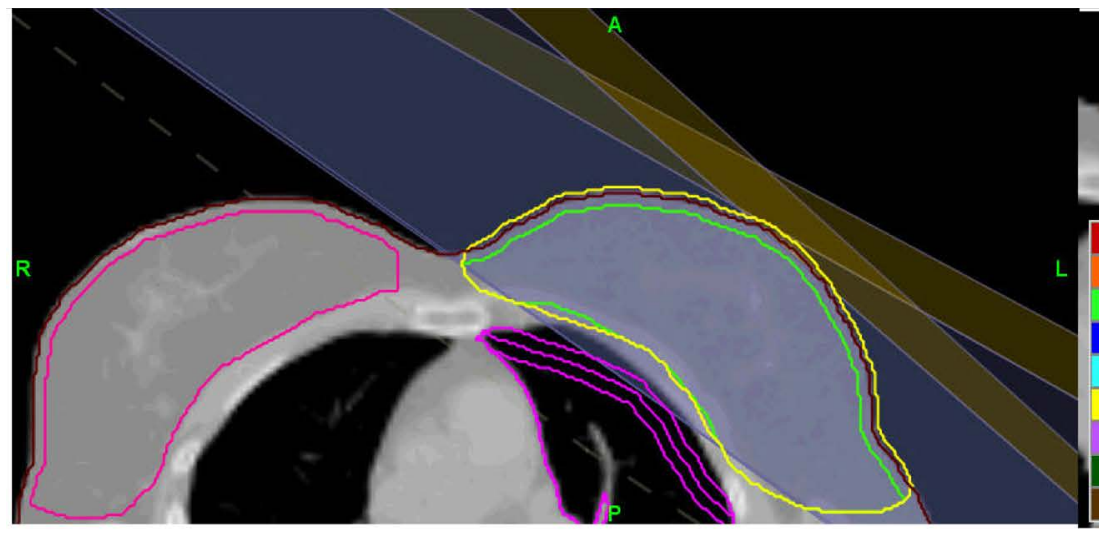

(a)

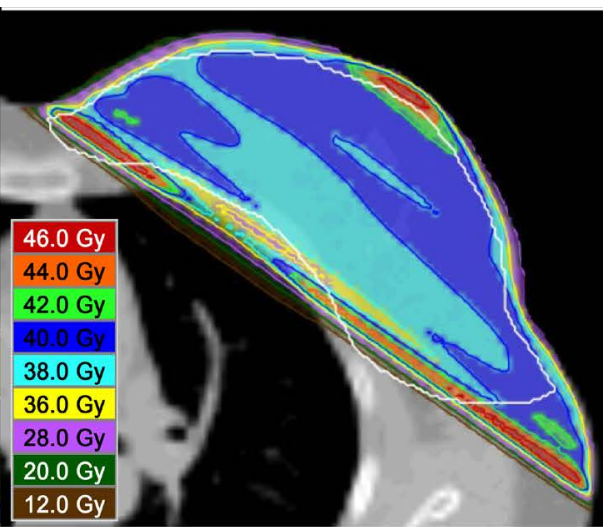

(b)

Figure 1. Illustration of contouring and beam arrangement used for planning and hot-spots in the plan. (a) Contralateral breast (pink), PTV (yellow), PTV_eval (green), and "LungHeartBlocking” structures (magenta). Two tangential beams (blue overlay) are used for WBI with 3 flash leaves (yellow overlay). Beam does not fully cover PTV due to the contralateral breast block structure and LungHeartBlocking structure used; (b) The plan created by tomotherapy TPS contains hot spots at the posterior edge of the tangential beams and near the tip of the breast. 
to achieve the desired OAR sparing, small areas of the breast_PTV may be completely blocked from the primary radiation.

Our observations led us to believe that the 3DCRT optimization algorithm, designed to achieve excellent dose homogeneity within the target, was causing the severe hot spots as illustrated in Figure 2. Figure 2(a) shows a hypothetical 1D dose profile perpendicular to the beam direction. Let's assume due to the existence of a blocking structure, a small portion of the PTV received 0 Gy while the rest of the PTV received the prescription dose of 60 Gy. Figure 2(b) illustrates another hypothetical dose profile after the edge leave opened for longer time. It created a hotspot of $80 \mathrm{~Gy}(133 \%)$ while increased the cold spot dose by 5 Gy through scattering. Tomotherapy's optimization, like most other treatment planning software optimization engines, uses quadratic function in the objective function. In the simplest form, the optimization can be expressed as $\arg \min \left(\left|D-D_{p}\right|^{2}\right)$, where $D$ is the dose and $D_{p}$ is the prescription dose. The objective value for dose distribution in Figure 2(b) is actually lower than Figure 2 (a), meaning that the optimization engine will choose Figure 2(b) as the better plan despite the severe hot-spot.

In order to illustrate the observed hot spot problem with the tomotherapy 3DCRT software optimization seen in clinical use, eight whole breast irradiation cases (4 left-sided and 4 right-sided) were randomly selected and planned using the RTOG 1005 target volume and OAR specifications. Specifically, breast CTV was contoured based on RTOG breast cancer atlas. Pectorialis muscles, chest wall and first $5 \mathrm{~mm}$ tissue under the skin were excluded from breast CTV. Breast_PTV took $7 \mathrm{~mm}$ expansion of the breast_CTV. An evaluation structure PTV_eval was created that excludes the part of the PTV that extended outside patient or into boney thorax and lungs and first $5 \mathrm{~mm}$ of tissue under the skin. During the treatment planning study, we tested the use of PTV_eval as planning

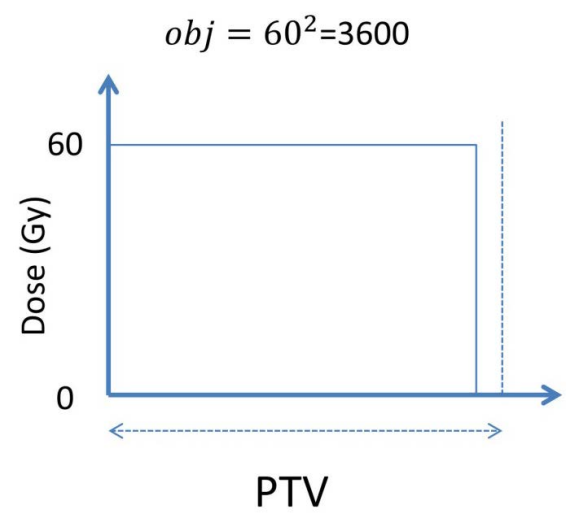

(a)

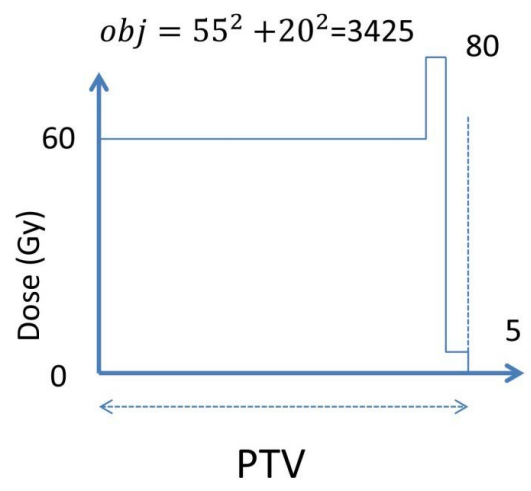

(b)

Figure 2. Illustration of how optimization engine creates hot-spot as a result of trying to reduce the cold spot. (a) 1D dose profile of a PTV which met the prescription dose of 60 Gy except that a cold near the edge got $0 \mathrm{~Gy}$; (b) 1D dose profile of the PTV with the dose at the edge increased to $80 \mathrm{~Gy}$ and the cold spot receives $5 \mathrm{~Gy}$. (b) will be favored by optimization engine because of the lower objective value. 
target instead of the breast_PTV structure.

Treatment planning was performed with TomoTherapy TomoHD V1.2 planning station (Accuray Inc. Madison, WI) powered with VoLO technology [24] [25] [26] [27]. A $2.5 \mathrm{~cm}$ jaw width and "fine" final dose calculation grid was used. Two tangential beams with 3 flash leaves were used to create WBI plans using the RTOG-1005 hypofractionation arm prescription. The prescribed dose was 40 Gy in 15 fractions. Since this study is centered on the hot-spot in the WBI plan, the boost plans were not included in our discussion. Complete blocks were applied to the contralateral breast and one of the "LungHeartBlocking" structures. If the plan did not meet the RTOG-1005 "ideal” constraints, a different "LungHeartBlocking" structure or a slightly different beam angle was used. When the ideal constraint could not be met, the goal was lowered to the acceptable constraint. In most conditions, lowering the PTV_eval coverage from 95\% receives 38 Gy to $90 \%$ receives 36 Gy was sufficient. The detailed protocol constraints are listed in Table 1 . According to our experience, the OAR constraints that are difficult to meet include heart mean dose, ipsilateral lung V16, contralateral lung V4, and contralateral breast Dmax.

In addition to the use of blocking structures and adjustment of percentage of target coverage, the treatment planning software also allows the user to adjust "compensation" and "normal tissue homogeneity". The compensation controls the modulation factor (a measure of intensity-modulation) and have two choices,

Table 1. RTOG 1005 ARM II target and OAR dose constraints for whole breast irradiation plan [23].

\begin{tabular}{|c|c|c|}
\hline Contour & Ideal constraints & Acceptable constraints \\
\hline \multirow{4}{*}{$\begin{array}{c}\text { Breast PTV eval } \\
\text { (whole breast volume) }\end{array}$} & $\mathrm{V} 38 \geq 95 \%$ & $\mathrm{~V} 36 \geq 90 \%$ \\
\hline & $\mathrm{V} 43.2 \leq 50 \%$ & $\mathrm{~V} 44.8 \leq 50 \%$ \\
\hline & $\mathrm{Dmax}<46 \mathrm{~Gy}$ & Dmax $<48$ Gy \\
\hline & $0.95 \leq \mathrm{CI} \leq 2.0$ (optional) & $0.85 \leq \mathrm{CI} \leq 3.0$ (optional) \\
\hline \multirow{2}{*}{ Contralateral breast } & $\mathrm{V} 1.44 \leq 5 \%$ & $\mathrm{~V} 2.4 \leq 5 \%$ \\
\hline & $\mathrm{Dmax}<2.4 \mathrm{~Gy}$ & $\mathrm{Dmax}<3.84 \mathrm{~Gy}$ \\
\hline \multirow{3}{*}{ Ipsilateral lung } & $\mathrm{V} 16 \leq 15 \%$ & $\mathrm{~V} 16 \leq 20 \%$ \\
\hline & $\mathrm{V} 8 \leq 35 \%$ & $\mathrm{~V} 8 \leq 40 \%$ \\
\hline & $\mathrm{V} 4 \leq 50 \%$ & $\mathrm{~V} 4 \leq 55 \%$ \\
\hline Contralateral lung & $\mathrm{V} 4 \leq 10 \%$ & $\mathrm{~V} 4 \leq 15 \%$ \\
\hline \multirow{3}{*}{$\begin{array}{c}\text { Heart } \\
\text { (for right sided tumors) }\end{array}$} & $\mathrm{V} 16=0 \%$ & $\mathrm{~V} 20=0 \%$ \\
\hline & $\mathrm{V} 8 \leq 10 \%$ & $\mathrm{~V} 8 \leq 15 \%$ \\
\hline & Dmean $\leq 3.2 \mathrm{~Gy}$ & Dmean $\leq 4.0 \mathrm{~Gy}$ \\
\hline \multirow{3}{*}{$\begin{array}{c}\text { Heart } \\
\text { (for left sided tumors) }\end{array}$} & $\mathrm{V} 16 \leq 5 \%$ & $\mathrm{~V} 20 \leq 5 \%$ \\
\hline & $\mathrm{V} 8 \leq 30 \%$ & $\mathrm{~V} 8 \leq 35 \%$ \\
\hline & Dmean $\leq 3.2 \mathrm{~Gy}$ & Dmean $\leq 4.0 \mathrm{~Gy}$ \\
\hline Thyroid & $\mathrm{Dmax} \leq 0.96 \mathrm{~Gy}$ & $\mathrm{Dmax} \leq 1.44 \mathrm{~Gy}$ \\
\hline
\end{tabular}


high or low. The normal tissue homogeneity applies penalty to hot-spots in regions outside of the target structures. To demonstrate how various plan settings affect the hot-spots, four plans for each patient were created:

1) "PTV_eval LC + NTH" as baseline: PTV_eval as prescription target, "Low compensation (LC)" and the "normal tissue homogeneity (NTH)" checked.

2) "PTV_eval LC", differed from baseline plan in that the NTH were not checked.

3) "PTV_eval HC + NTH", differed from the baseline plan in that the "high compensation (HC)" was checked.

4) "PTV LC + NTH", uses the breast_PTV as prescription target with low compensation and normal tissue homogeneity checked.

All 4 plans for the same patient used the exactly same beam angle and flash leaves. The calculations were run at "normal" dose grid and the final doses were computed in "fine" dose grid.

Specific dose metrics of interest were hot-spot $\mathrm{D}_{\max }$ (defined as maximum dose in the patient volume), hot-spot volume (defined as volume outside of PTVs receiving more than $110 \%$ of prescription), ipsilateral lung $\mathrm{D}_{15 \%}$, contralateral lung $\mathrm{D}_{10 \%}$, heart $\mathrm{D}_{\text {mean }}$, contralateral breast $\mathrm{D}_{\max }$, breast PTV_eval Conformity Index (CI). The CI was defined according to RTOG-1005 as the volume covered by 95\% of the prescription isodose divided by the volume of the target PTV_eval. Wilcoxon signed-rank test was used to test the statistical significance of the observed difference.

In order to further validate our hypothesis that the tomotherapy 3DCRT software optimization algorithm was causing the hot spots we observed clinically, all 8 plans with a hot spot was manually modified by adjusting the edge MLCs in order to adjust the fluence at the beam edge. This involves a labor intensive process of identifying the delivery plan from the patient archive, modifying leaf events file, and restoring archive in TomoTherapy TPS for dose re-calculation. The posterior edge leaves open time were reduced by $15 \%$ in all 8 cases. No individual optimization was performed. The hot-spot $D_{\max }$ and volume and OAR dose for the modified plan was evaluated.

\section{Results}

Figure 3 shows the isodose distributions from the 4 planning strategies of a representative patient case. All 4 plans show hot-spots along the posterior field edge. In addition, hot-spots appear near the surface of the breast when breast_PTV is used as target. The width of the hot-spot along the posterior field edge indicates that it could be the result of a single leaf opening too long.

All RTOG-1005 criteria other than the hot-spot can be satisfied for all eight patients in all four planning modes. Table 2 summarized the dosimetric comparison between different planning modes. Using "PTV_eval LC + NTH" as baseline, other planning schemes were compared using the Wilcoxon signed-rank test and the $\mathrm{p}$ value is listed. There is little difference in target coverage and OAR 

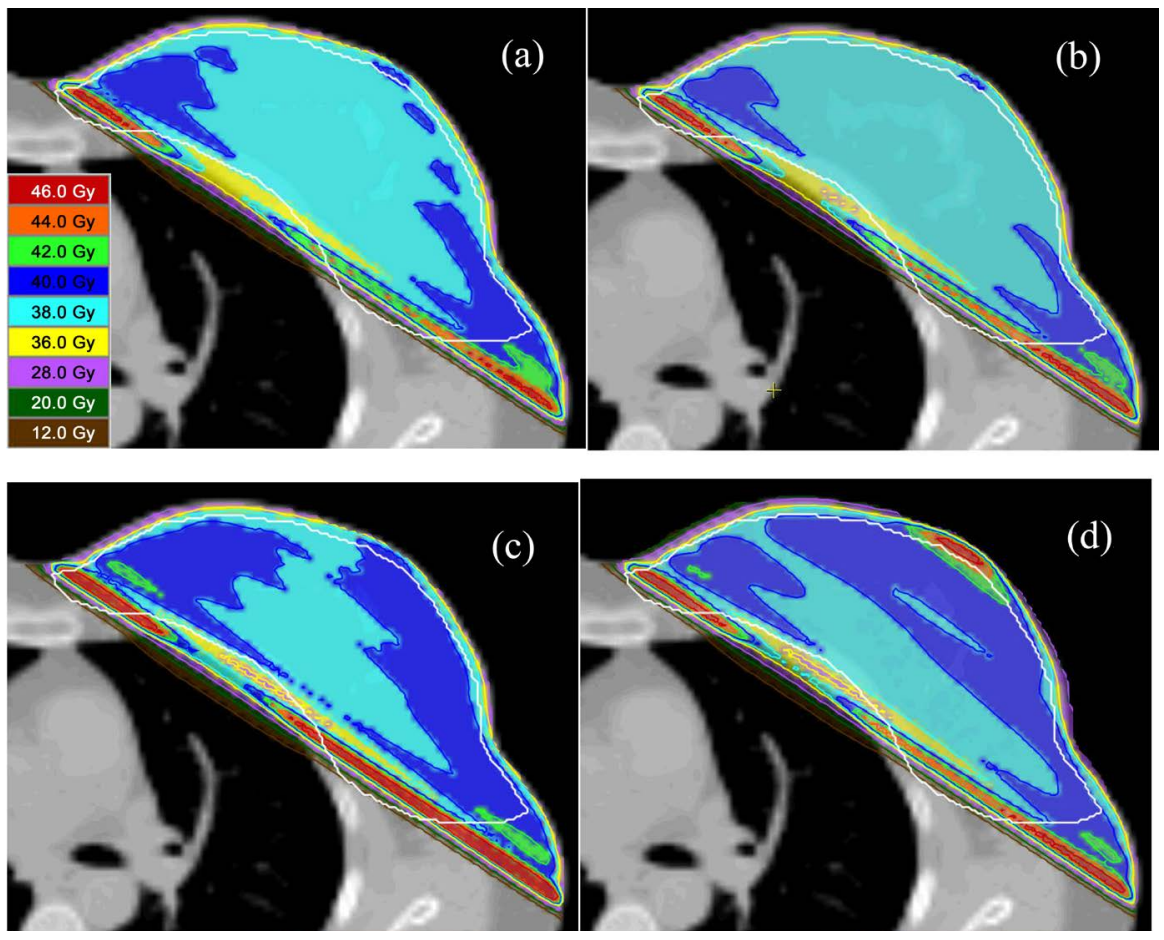

Figure 3. Isodose distribution of a representative case planned with (a) PTV_eval LC+NTH; (b) PTV_eval LC; (c) PTV_eval HC + NTH; and (d) PTV LC + NTH. PTV_eval was outlined in these figures for reference. Other structures and beam angles were illustrated on Figure 1.

Table 2. Dosimetric comparison between plans with different planning settings.

\begin{tabular}{|c|c|c|c|c|c|c|c|}
\hline & $\begin{array}{c}\text { PTVEval } \\
\text { LC + NTH }\end{array}$ & $\begin{array}{c}\text { PTVEval } \\
\text { LC }\end{array}$ & & $\begin{array}{c}\text { PTVEval } \\
\mathrm{HC}+\mathrm{NTH}\end{array}$ & & $\begin{array}{c}\text { PTV } \\
\text { LC + NTH }\end{array}$ & \\
\hline & Mean (SD) & Mean (SD) & $\mathrm{P}$ & Mean (SD) & $\mathrm{P}$ & Mean (SD) & $\mathrm{P}$ \\
\hline D_hotspot (Gy) & $48.5(1.6)$ & $49.6(2.5)$ & $<0.001$ & $58.6(3.4)$ & $<0.001$ & $50.2(1.7)$ & $<0.001$ \\
\hline V_hotspot (cc) & $30.4(25.2)$ & $44.5(35.5)$ & $<0.001$ & $71.8(45.6)$ & $<0.001$ & $56.6(39.6)$ & $<0.001$ \\
\hline Ipsilateral Lung D15 (Gy) & $5.2(3.5)$ & $5.3(3.5)$ & $<0.001$ & $5.7(3.9)$ & $<0.001$ & $5.4(3.8)$ & $>0.05$ \\
\hline Contrlat Lung D10 (Gy) & $0.3(0.1)$ & $0.4(0.1)$ & $>0.05$ & $0.3(0.1)$ & $>0.2$ & $0.3(0.1)$ & $>0.2$ \\
\hline Heart Dmean (Gy) & $1.3(0.9)$ & $1.3(0.9)$ & $<0.001$ & $1.4(0.9)$ & $<0.001$ & $1.3(0.9)$ & $<0.001$ \\
\hline Contrlat Breast Dmax (Gy) & $1.2(0.4)$ & $1.2(0.4)$ & $<0.001$ & $1.3(0.6)$ & $<0.001$ & $1.2(0.4)$ & $>0.2$ \\
\hline PTVeval CI & $1.4(0.2)$ & $1.5(0.2)$ & $>0.05$ & $1.4(0.2)$ & $>0.05$ & $1.5(0.2)$ & $>0.1$ \\
\hline PTV Dmean (Gy) & $39.4(0.5)$ & $39.8(0.6)$ & $>0.2$ & $39.9(0.5)$ & $>0.1$ & $39.3(0.5)$ & $<0.001$ \\
\hline
\end{tabular}

constraints for all 4 plan strategies. However, differences existed in hot-spot metrics with hot-spots appearing medial or lateral to the planning target volumes. The use of PTV_eval as target structure, together with low compensation and normal tissue homogeneity, produces maximum hot-spot of $48.5 \mathrm{~Gy}$ (121\% of prescription dose) and hot-spot volume of $30.4 \mathrm{cc}$, both the lowest among the 4 plans. Switching off the normal tissue uniformity slightly increases the maximum hot-spot to 49.6 Gy (124\% of prescription dose) and hot-spot volume to 
$44.5 \mathrm{cc}$. Switching on normal tissue homogeneity with high compensation produced a dramatic increase of maximum hot-spot to 58.6 Gy or $146.5 \%$ of prescription dose and hot-spot volume to $71.8 \mathrm{cc}$. Finally, using breast_PTV as target structure with low compensation and normal tissue homogeneity produces a moderate increase of maximum hot-spot to 50.2 Gy or $125.5 \%$ and hot-spot volume to $56.6 \mathrm{cc}$. Figure 4 shows the maximum hot-spot dose and hot-spot volume under different planning strategies for the 8 patients in this study. The normal tissue homogeneity does not seem to produce a big difference in both the hot-spot dose and volume for the baseline plan with low compensation. On the other hand, the use of high modulation factor significantly increases the hot-spot dose $(p=0.00005)$ and volume $(p=0.006)$. Compared with baseline, the use of breast_PTV as the target structure also significantly increases the hot-spot volume $(\mathrm{p}=0.007)$ and dose $(\mathrm{p}=0.003)$.

Figure 5(a) shows the leaf open sinogram that produces the baseline plan shown in Figure 3(a). The first 80 projections shows the leaf open sinogram for the Left Posterior Oblique (LPO) field and the next 80 projections shows the leaf open sinogram for the Left Anterior Oblique (LAO) field. Figure 5(b) shows the leaf open time for projection 30. Both figures confirm that the MLC leaf at the posterior field edge has much longer open time. Figure $5(\mathrm{c})$ shows the modified sinogram after a simple strategy to reduce the open time of all posterior edge leaves by $15 \%$. And Figure 5(d) shows the isodose distribution after the modification. Comparing with Figure 3(a), the hot-spot is significantly reduced while the target coverage maintains at 95\% PTV_eval receives $38 \mathrm{~Gy}$. The maximum hot-spot dose drops from $120 \%$ to $113 \%$ and the hot-spot volume drops from 34 cc to $5.4 \mathrm{cc}$.

Table 3 summarizes the improvement in dosimetry after manual modification of the plan sinogram. Wilcoxon signed-rank test was used to evaluate the statistical significance of the change. Both the volume and the magnitude of hot spot
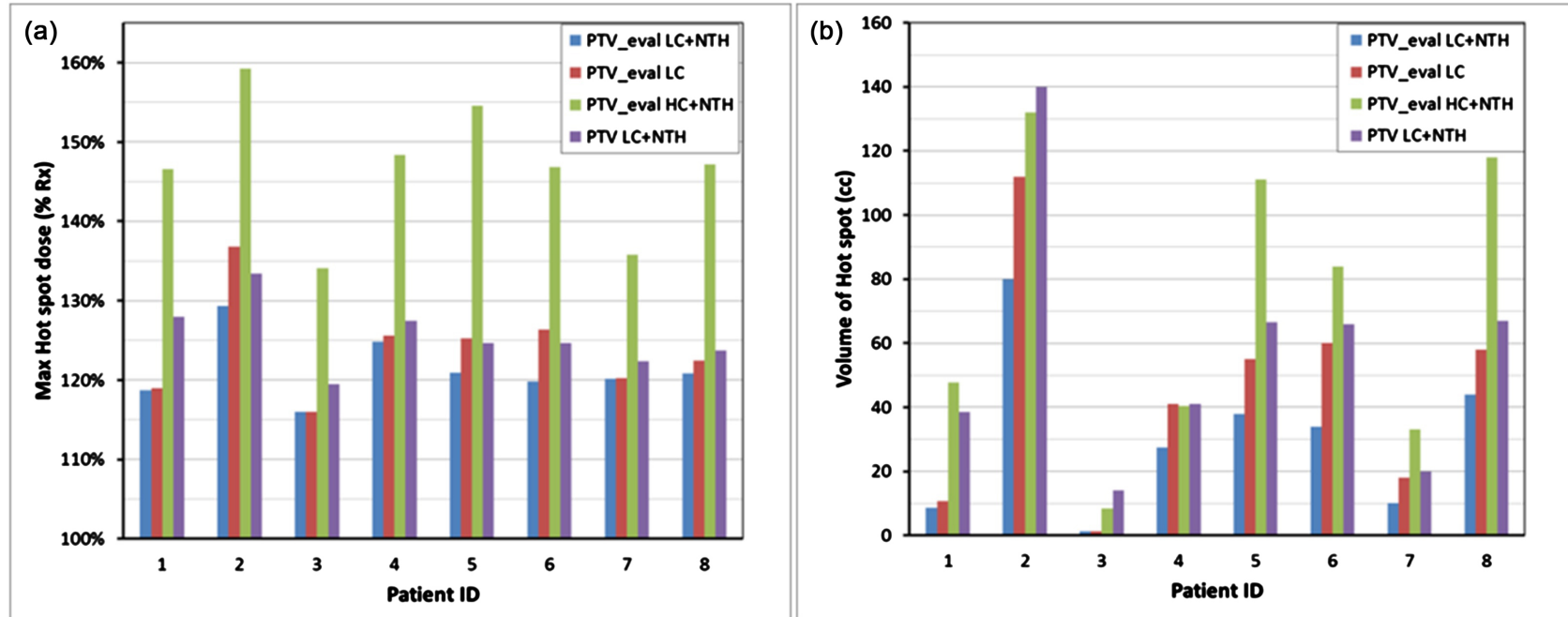

Figure 4. (a) Maximum hot-spot dose expressed as percentage of prescription and (b) volume of hot-spot (defined as the volume of tissue receiving greater than $110 \%$ of prescription) with each planning methods for the 8 patients used in this study. 


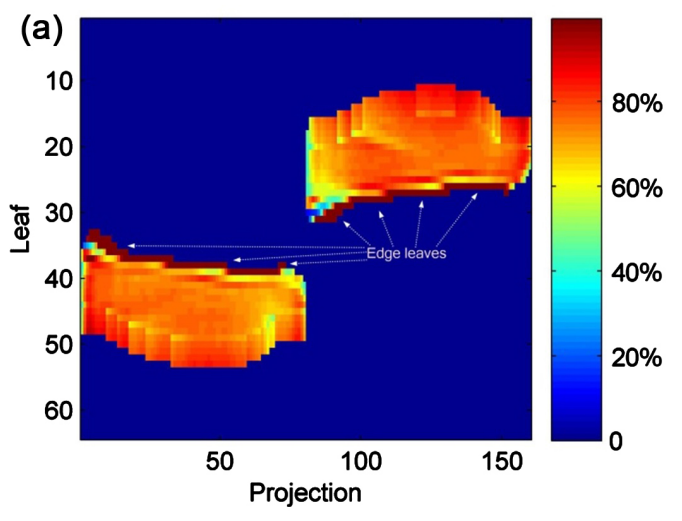

(c)

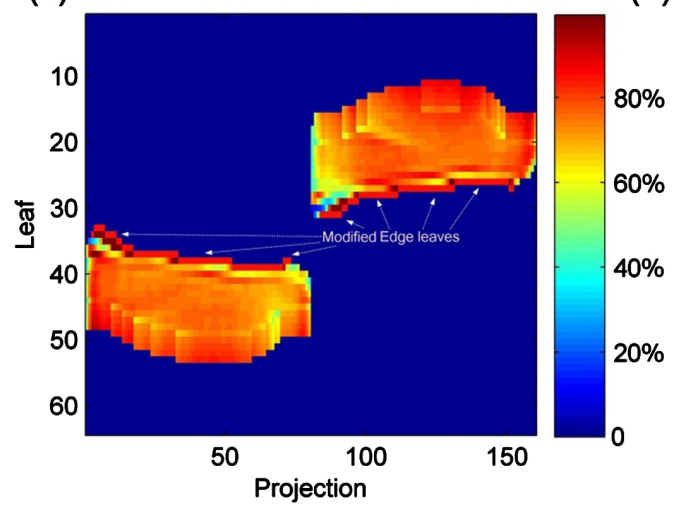

(d)
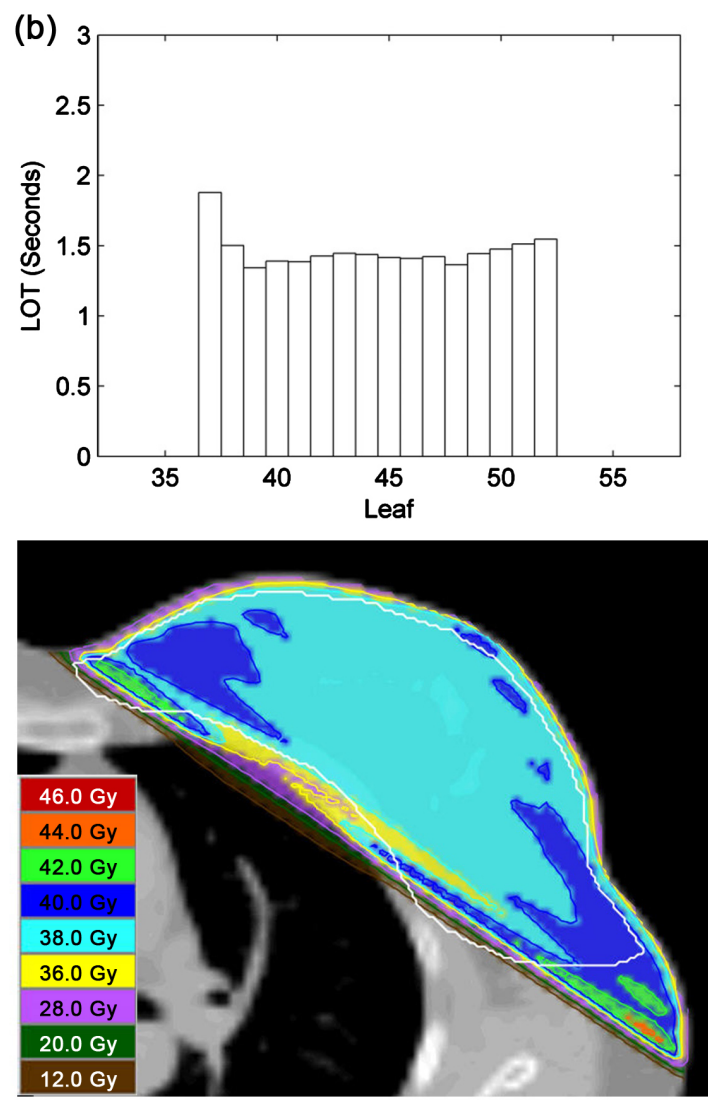

Figure 5. Reducing hot-spot by manually modifying the delivery plan sinogram. (a) original sinogram that produced Figure 3(a); (b) leaf open time (LOT) for projection 30; (c) modified sinogram after posterior edge leaves open time were reduced by $15 \%$; and (d) the dose from modified sinogram. The maximum point dose in normal tissue reduced from $48 \mathrm{~Gy}$ to $45 \mathrm{~Gy}$ and the volume received greater than $44 \mathrm{~Gy}$ reduced from $34 \mathrm{cc}$ to $5.4 \mathrm{cc}$ comparing with Figure 3(a).

Table 3. Dosimetric comparison after manual modification of the plan sinogram.

\begin{tabular}{cccc}
\hline & $\begin{array}{c}\text { PTVEval } \\
\text { LC + NTH }\end{array}$ & $\begin{array}{c}\text { Manual } \\
\text { Adjustment }\end{array}$ & \\
\hline Mean (SD) & Mean (SD) & p \\
D_hotspot (Gy) & $48.5(1.6)$ & $44.4(1.6)$ & $<0.001$ \\
V_hotspot (cc) & $30.4(25.2)$ & $6.1(10.5)$ & $<0.001$ \\
Ipsilateral Lung D15 (Gy) & $5.2(3.5)$ & $5.1(3.3)$ & $>0.1$ \\
Contrlat Lung D10 (Gy) & $0.3(0.1)$ & $0.3(0.1)$ & $>0.1$ \\
Heart Dmean (Gy) & $1.3(0.9)$ & $1.1(0.9)$ & $<0.001$ \\
Contrlat Breast Dmax (Gy) & $1.2(0.4)$ & $1.2(0.3)$ & $>0.1$ \\
PTVeval CI & $1.4(0.2)$ & $1.3(0.2)$ & $<0.001$ \\
PTV Dmean (Gy) & $39.4(0.5)$ & $38.9(0.3)$ & $<0.001$ \\
\hline
\end{tabular}

were significantly improved $(\mathrm{p}<0.001)$. OAR doses were slightly better as well although most changes did not have any statistical significance $(\mathrm{p}>0.1)$. PTV mean dose has a statistically significant reduction $(\mathrm{p}<0.001)$, although the 
amount of reduction is small (1.25\%). Figure 6 shows the reduction in hot spot dose and volume for each case after plan sinogram modification. Five of the eight cases successfully reduced the hot spot dose to within $110 \%$ of the prescription. The remaining cases also have respectable reduction in hot spot dose and volume.

\section{Discussion}

With traditional tangential breast treatment on LINAC, the extent of the treatment field is determined by the markers placed around the breast. The target volume is not drawn, rather it is assumed to be covered entirely by the tangential treatment field. The contralateral breast is not usually drawn either. Recently, RTOG requires both the target volume and contralateral breast to be drawn for treatment planning [23]. In particular, the medial border of the breast should start at the sternal-costal junction. Additionally, due to the concern of the secondary cancer risk in the contralateral breast [28], the RTOG1005 imposes a strict contralateral breast dose constraint (max dose 2.4 Gy ideal, 3.84 Gy acceptable). In order to meet those constraints, the extent of the treatment field can be limited to exclude a small section of the target volume in order to achieve better OAR sparing. This, in turn, creates conditions for the hot spots to arise. These hot spots may lead to both acute and late toxicities.

We noted that several studies investigating the feasibility of using static tomotherapy on breast treatment does not suffer from hot spot issue [29] [30] [31]. However, this is likely due to the difference in planning constraint and contouring guideline used. In Yadav et al.'s study, the contralateral breast maximum dose reaches 19.98 Gy on average [31], In Reynders et al.'s study, both target and contralateral breast is under-contoured in the medial side, as shown in their supplementary data [30]. These approaches may enable them to have complete coverage of the target volume by the primary tangential beam. Hashimoyo, et al.
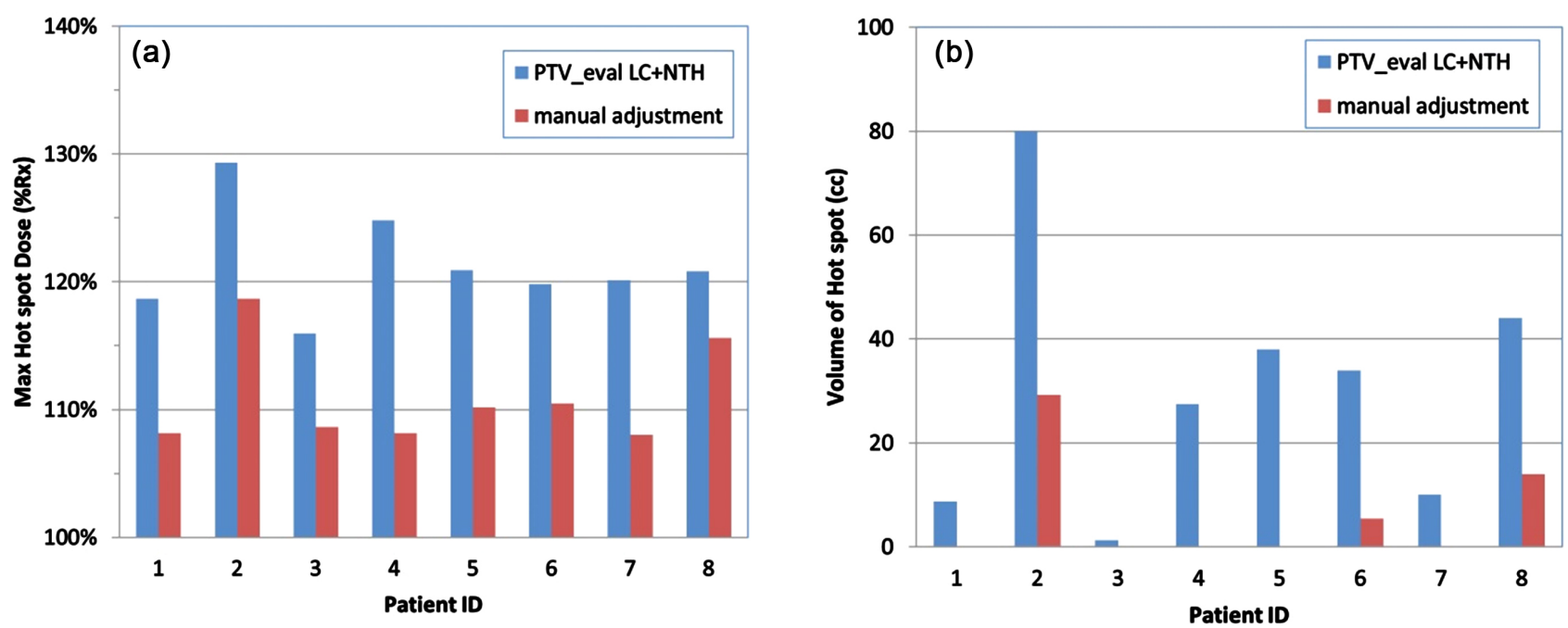

Figure 6. Reduction of (a) hot-spot dose and (b) volume of hot-spot after manually reduce posterior leaf open time by $15 \%$ for the 8 patients used in this study. 
showed the great plan quality achieved by TomoDirect IMRT mode 29 . The use of IMRT mode allowed the planner to adjust dose-volume histogram points to control hot spots. Therefore, these studies did not undermine the significance of findings in our study.

Similar clinically unacceptable hot spots that we and others have observed when using TomoDirect 3DCRT for whole breast irradiation were reproducible in the patient studies we performed. We observed that increased modulation increases these hot spots further. We also observed that manually reducing the opening time of the posterior edge leaf reduced the magnitude and volume of the hot spot. These two observations provide evidence that the software dose optimization algorithm is contributing to the hot spots.

While our study suggests that adjusting the opening time of the posterior edge MLC in TomoDirect 3DCRT opposed tangential beams in breast cancer can improve dose homogeneity, performance of this task manually is a cumbersome labor-intensive process that required us to archive and alter the patient plan. We were only able to guess how much of an adjustment to make without the immediate feedback of the dose information. Only after restoring the plan archive to tomotherapy database can we perform the dose calculation to evaluate the effect of our adjustment. Therefore, it was not feasible for us to optimize the magnitude of the adjustment, and determine whether multiple MLCs require adjustment, or test clinical implementation of such adjustments. Despite these limitations, we believe our study demonstrates that the optimization algorithm inherent to the tomotherapy treatment planning software is not optimizing the MLCs in a way that makes the most sense clinically and that this software has room for improvement.

Based on our finding, another possible solution is to create a different target structure for planning that excludes the regions that is not covered by the primary beam due to the blocking, similar to the structure proposed by Fields et al [10]. However, the extent of the new target structure depends on the tangential angle selected and there are no existing functions in TPS to create this structure. Therefore, we have not tested this solution. We also believe that while this solution will very likely reduce the hot spots reported in this study, it does not change the fact that the user has limited control on the dose distribution created by the tomotherapy 3DCRT mode. Therefore, creating a forward-planning interface that allows user to fine tune the fluence adds a much needed flexibility for the tomotherapy 3DCRT mode.

Our study does not exclude the possibility that the relatively soft $6 \mathrm{MV}$ photon beam inherent to tomotherapy is a contributing factor to the hot spots as others have suggested. Nevertheless, our study demonstrates that the beam energy is not the major culprit.

Our study demonstrates that a flawed software optimization algorithm is at least a contributing factor in causing the clinically unacceptable hot spots outside the planning target volumes in breast cancer patients planned with opposed 
tangential beams using 3DCRT TomoDirect. This is likely limiting the use of 3DCRT TomoDirect in treatment of breast cancer in other facilities, as it has at our own facility. Facilities that have alternate equipment may prefer to treat their patients who need whole breast irradiation on the alternate equipment. Facilities that do not have alternate equipment may be compelled to treat breast cancer patients using IMRT, although this methodology is much more expensive than 3DCRT and insurance approval may not be granted for its use, or transfer patients to other facilities to be treated with other equipment. It also seems likely that the issues we have demonstrated in this study with the use of opposed tangential 3DCRT TomoDirect beams in breast cancer would be observed, as we have ourselves, in other situations where targets were not fully covered by the primary beam. In these other situations we have usually been able to overcome this problem by adding other beam angles, which is not safe to do in breast cancer as doing this increases the dose to the lungs, the heart and stomach in left breast cancer, and the liver in right breast cancer.

\section{Conclusion}

In this study, we investigated the hot-spots in tomotherapy static-beam 3DCRT WBI plans. While we found that these plans resulted in excellent sparing of organs at risk, the planning algorithm resulted in clinically unacceptable hot-spots at the posterior field edges. The origin of those hot-spots was analyzed and explained. Further study showed that it was possible to reduce these hot-spots by adjusting the delivery plan. We believe that static-beam 3DCRT tomotherapy could be modified by allowing the user to adjust fluences in the beam's eye view. Such an improvement could make tomotherapy a good modality for treating breast cases using TD 3DCRT.

\section{Conflicts of Interest}

The authors declare no conflicts of interest regarding the publication of this paper.

\section{References}

[1] Arriagada, R., Le, M.G., Rochard, F. and Contesso, G. (1996) Conservative Treatment versus Mastectomy in Early Breast Cancer: Patterns of Failure with 15 Years of Follow-Up Data. Institut Gustave-Roussy Breast Cancer Group. Journal of Clinical Oncology, 14, 1558-1564. https://doi.org/10.1200/JCO.1996.14.5.1558

[2] Blichert-Toft, M., Nielsen, M., During, M., et al. (2008) Long-Term Results of Breast Conserving surgery vs. Mastectomy for Early Stage Invasive Breast Cancer: 20-year Follow-Up of the Danish Randomized DBCG-82TM Protocol. Acta Oncologica, 47, 672-681. https://doi.org/10.1080/02841860801971439

[3] Blichert-Toft, M., Rose, C., Andersen, J.A., et al. (1992) Danish Randomized Trial Comparing Breast Conservation Therapy with Mastectomy: Six Years of Life-Table Analysis. Danish Breast Cancer Cooperative Group. J Natl Cancer Inst Monogr, 19-25.

[4] Fisher, B., Anderson, S., Bryant, J., et al. (2002) Twenty-Year Follow-Up of a Ran- 
domized Trial Comparing Total Mastectomy, Lumpectomy, and Lumpectomy plus Irradiation for the Treatment of Invasive Breast Cancer. The New England Journal of Medicine, 347, 1233-1241. https://doi.org/10.1056/NEJMoa022152

[5] Poggi, M.M., Danforth, D.N., Sciuto, L.C., et al. (2003) Eighteen-Year Results in the Treatment of Early Breast Carcinoma with Mastectomy versus Breast Conservation Therapy: The National Cancer Institute Randomized Trial. Cancer, 98, 697-702. https://doi.org/10.1002/cncr.11580

[6] van Dongen, J.A., Voogd, A.C., Fentiman, I.S., et al. (2000) Long-Term Results of a Randomized Trial Comparing Breast-Conserving Therapy with Mastectomy: European Organization for Research and Treatment of Cancer 10801 Trial. Journal of the National Cancer Institute, 92, 1143-1150.

https://doi.org/10.1093/jnci/92.14.1143

[7] Veronesi, U., Cascinelli, N., Mariani, L., et al. (2002) Twenty-Year Follow-Up of a Randomized Study Comparing Breast-Conserving Surgery with Radical Mastectomy for Early Breast Cancer. The New England Journal of Medicine, 347, 1227-1232. https://doi.org/10.1056/NEJMoa020989

[8] Anderson, S.J., Wapnir, I., Dignam, J.J., et al. (2009) Prognosis after Ipsilateral Breast Tumor Recurrence and Locoregional Recurrences in Patients Treated by Breast-Conserving Therapy in Five National Surgical Adjuvant Breast and Bowel Project Protocols of Node-Negative Breast Cancer. Journal of Clinical Oncology, 27, 2466-2473. https://doi.org/10.1200/JCO.2008.19.8424

[9] Wapnir, I.L., Anderson, S.J., Mamounas, E.P., et al. (2006) Prognosis after Ipsilateral Breast Tumor Recurrence and Locoregional Recurrences in Five National Surgical Adjuvant Breast and Bowel Project Node-Positive Adjuvant Breast Cancer Trials. Journal of Clinical Oncology, 24, 2028-2037. https://doi.org/10.1200/JCO.2005.04.3273

[10] Fields, E.C., Rabinovitch, R., Ryan, N.E., Miften, M. and Westerly, D.C. (2013) A Detailed Evaluation of TomoDirect 3DCRT Planning for Whole-Breast Radiation Therapy. Medical Dosimetry, 38, 401-406. https://doi.org/10.1016/j.meddos.2013.04.008

[11] Barnett, G.C., Wilkinson, J., Moody, A.M., et al. (2009) A Randomised Controlled Trial of Forward-Planned Radiotherapy (IMRT) for Early Breast Cancer: Baseline Characteristics and Dosimetry Results. Radiotherapy and Oncology, 92, 34-41. https://doi.org/10.1016/j.radonc.2009.03.003

[12] Fogliata, A., Nicolini, G., Alber, M., et al. (2005) IMRT for Breast. A Planning Study. Radiotherapy and Oncology, 76, 300-310. https://doi.org/10.1016/j.radonc.2005.08.004

[13] Schubert, L.K., Gondi, V., Sengbusch, E., et al. (2011) Dosimetric Comparison of Left-Sided Whole Breast Irradiation with 3DCRT, Forward-Planned IMRT, Inverse-Planned IMRT, Helical Tomotherapy, and Topotherapy. Radiotherapy and Oncology, 100, 241-246. https://doi.org/10.1016/j.radonc.2011.01.004

[14] Freedman, G.M., Anderson, P.R., Li, J., et al. (2006) Intensity Modulated Radiation Therapy (IMRT) Decreases Acute Skin Toxicity for Women Receiving Radiation for Breast Cancer. American Journal of Clinical Oncology, 29, 66-70. https://doi.org/10.1097/01.coc.0000197661.09628.03

[15] Pignol, J.P., Olivotto, I., Rakovitch, E., et al. (2008) A Multicenter Randomized Trial of Breast Intensity-Modulated Radiation Therapy to Reduce Acute Radiation Dermatitis. Journal of Clinical Oncology, 26, 2085-2092. https://doi.org/10.1200/JCO.2007.15.2488

[16] Dayes, I., Rumble, R.B., Bowen, J., et al. (2012) Intensity-Modulated Radiotherapy 
in the Treatment of Breast Cancer. Clinical Oncology, 24, 488-498. https://doi.org/10.1016/j.clon.2012.05.003

[17] Smith, B.D., Pan, I.W., Shih, Y.C., et al. (2011) Adoption of Intensity-Modulated Radiation Therapy for Breast Cancer in the United States. Journal of the National Cancer Institute, 103, 798-809. https://doi.org/10.1093/jnci/djr100

[18] Mackie, T.R., Holmes, T., Swerdloff, S., et al. (1993) Tomotherapy: A New Concept for the Delivery of Dynamic Conformal Radiotherapy. Medical Physics, 20, 1709-1719. https://doi.org/10.1118/1.596958

[19] Oliver, M., Ansbacher, W. and Beckham, W.A. (2009) Comparing Planning Time, Delivery Time and Plan Quality for IMRT, Rapid Arc and Tomotherapy. Journal of Applied Clinical Medical Physics, 10, 117-131. https://doi.org/10.1120/jacmp.v10i4.3068

[20] Jones, R., Yang, W., Read, P. and Sheng, K. (2011) Radiation Therapy of Post-Mastectomy Patients with Positive Nodes Using Fixed Beam Tomotherapy. Radiotherapy and Oncology, 100, 247-252. https://doi.org/10.1016/j.radonc.2011.05.004

[21] Fraass, B.A., Steers, J.M., Matuszak, M.M. and McShan, D.L. (2012) Inverse-Optimized 3D Conformal Planning: Minimizing Complexity while Achieving Equivalence with Beamlet IMRT in Multiple Clinical Sites. Medical Physics, 39, 3361-3374. https://doi.org/10.1118/1.4709604

[22] Borca, V.C., Franco, P., Catuzzo, P., et al. (2012) Does TomoDirect 3DCRT Represent a Suitable Option for Post-Operative Whole Breast Irradiation? A Hypothesis-Generating Pilot Study. Radiation Oncology, 7, 211.

https://doi.org/10.1186/1748-717X-7-211

[23] RTOG1005: A Phase III Trial of Accelerated Whole Breast Irradiation with Hypofractionation plus Concurrent Boost versus Standard Whole Breast Irradiation plus Sequential Boost for Early-Stage Breast Cancer.

http://www.rtog.org/clinicaltrials/protocoltable/studydetails.aspx?study=1005

[24] Chen, Q., Chen, M. and Lu, W. (2011) Ultrafast Convolution/Superposition Using Tabulated and Exponential Kernels on GPU. Medical Physics, 38, 1150-1161. https://doi.org/10.1118/1.3551996

[25] Chen, Q., Lu, W., Chen, Y., et al. (2012) Validation of GPU Based TomoTherapy Dose Calculation Engine. Medical Physics, 39, 1877-1886. https://doi.org/10.1118/1.3693057

[26] Lu, W. (2010) A Non-Voxel-Based Broad-Beam (NVBB) Framework for IMRT Treatment Planning. Physics in Medicine \& Biology, 55, 7175-7210. https://doi.org/10.1088/0031-9155/55/23/002

[27] Lu, W. and Chen, M. (2010) Fluence-Convolution Broad-Beam (FCBB) Dose Calculation. Physics in Medicine \& Biology, 55, 7211-7229.

https://doi.org/10.1088/0031-9155/55/23/003

[28] Stovall, M., Smith, S.A., Langholz, B.M., et al. (2008) Dose to the Contralateral Breast from Radiotherapy and Risk of Second Primary Breast Cancer in the WECARE Study. International Journal of Radiation Oncology, Biology, Physics, 72, 1021-1030. https://doi.org/10.1016/j.ijrobp.2008.02.040

[29] Hashimoyo, H., Omura, M., Matsui, K., et al. (2015) Tangent Field Technique of TomoDirect Improves Dose Distribution for Whole-Breast Irradiation. Journal of Applied Clinical Medical Physics, 16, 5369.

[30] Reynders, T., Tournel, K., De Coninck, P., et al. (2009) Dosimetric Assessment of Static and Helical TomoTherapy in the Clinical Implementation of Breast Cancer Treatments. Radiotherapy and Oncology, 93, 71-79. 
https://doi.org/10.1016/j.radonc.2009.07.005

[31] Yadav, P., Yan, Y., Ignatowski, T. and Olson, A. (2017) Dosimetric Aspects of Breast Radiotherapy with Three-Dimensional and Intensity-Modulated Radiotherapy Helical Tomotherapy Planning Modules. Medical Dosimetry, 42, 42-46. https://doi.org/10.1016/j.meddos.2016.11.001 